\title{
A method for the retrospective estimation of the individual respiratory intake of a highly and a poorly metabolising solvent during rest and physical exercise
}

\author{
J J G OPDAM, J F J SMOLDERS \\ From the Coronel Laboratory, Faculty of Medicine, University of Amsterdam, 1105 AZ Amsterdam, \\ The Netherlands
}

\begin{abstract}
A method for the retrospective estimation of the individual respiratory intake was tested. The method is based on system dynamics. Subjects were exposed simultaneously to the poorly metabolising solvent tetrachloroethene (PER, perchloroethylene) and the highly metabolising trichloroethene (TRI) at rest, 30, and 65 watt physical exercise. The time courses of the alveolar concentration $\left(\mathrm{C}_{\mathrm{alv}}\right)$ of both PER and TRI were measured. A retrospective estimation of the individual intake of PER could be carried out up to 400 hours after exposure with 10-20\% accuracy, irrespective of the level of exercise. The estimates of the intake of TRI are less accurate. The $\mathrm{C}_{\mathrm{alv}}$ in the 1-15 hours postexposure permits the estimation of the intake of TRI within a mean error of $25 \%$ for most subjects. For men the method may be applied up to 48 hours after exposure within $20 \%$ error. For women the intake estimates showed a poor accuracy with the use of $\mathrm{C}_{\mathrm{alv}}$ beyond the day of exposure.
\end{abstract}

Biological monitoring programs should permit the detection of differences in individual uptake rather than in external exposure. ${ }^{1}$ To make a retrospective estimate of the external exposure at a fixed sampling point, relations have been established between external exposure and biological measures such as the concentration of solvents or metabolites in exhaled breath or biological fluids. ${ }^{2-4}$

Different individual minute volumes of ventilation and kinetics (distribution and elimination) may cause a large interindividual variability in biological measures at the same level of exposure. Up to now the estimation of the level of external exposure from biological media requires strict sampling protocols; nevertheless on an individual basis these estimates are mostly poor. In a previous paper it was pointed out that the kinetics and the rate of functional intake (RFI) are independent variables which determine the timecourse of the blood solvent/metabolite concentration and the rate of uptake. Therefore, one should estimate the RFI rather than the rate of uptake. ${ }^{5}$

Linear system dynamics studies the relation between the rate of input and the kinetic response. In the case of pharmacokinetics or toxicokinetics the

Accepted 18 April 1988 rate of input may be the rate of the respiratory, oral, dermal, metabolic or intravenous input and the response may be a kinetic response such as concentration time curve of the parent compound or its metabolites. The relation between input and response is a convolution integral in which the unit impulse response plays a central part. When two of the three (rate of input, kinetic response, or unit impulse response) are known the third may be calculated with a suitable method for convolution or deconvolution. ${ }^{6-10}$

The unit impulse response is an agent specific individual characteristic; it describes implicitly the complete kinetics of a parent compound or its metabolite in an individual subject. This means that all processes such as resorption, elimination, and metabolism determine the shape of the impulse response curve.

In the biological monitoring of solvent vapours both the individual rate of respiratory input and the individual kinetics are not usually known. The possibility of finding an accurate, retrospective individual RFI depends on the knowledge of the individual kinetics (unit impulse response). Furthermore, intraindividual differences in the kinetics of solvents and the metabolites also should not be neglected. 
The purpose of this study was to test a method based on linear system dynamics for the estimation of the individual rate of respiratory input during experimental exposure to a poorly and a highly metabolising organic solvent during rest and during physical exercise.

Subjects were exposed two or three times simultaneously to tetrachloroethene (PER) and trichloroethene (TRI) vapour under conditions of rest and physical exercise. For both PER and TRI the concentration in alveolar air was measured which is representative for the concentration in mixed venous blood."

\section{Theoretical background}

The body may be considered as a dynamic system composed of several subsystems. An input (amount/ time) of an agent or metabolite is administered into an input subsystem-usually blood-through the respiratory, oral, dermal, intravenous, peritoneal, or metabolic route; it may be regarded as a perturbation of the whole system. The system reacts on this with one or more kinetic responses in one or more subsystems; the kinetic responses, for example, are the concentration/time profiles of agent or metabolites in blood, exhaled breath, organ/tissues, urine, and saliva.

In system dynamics the operator which transforms the input into a kinetic response is considered to be the most important. The relation between input and response is a convolution integral in which a weighting function-that is, unit impulse response-weights past values of the input to give the present value of the response. The use of system dynamics is based on the following assumptions:

(1) The system is lumped, rather than distributed; its behaviour may be described over a finite number of points of time.

(2) The system is linear so that the principle of superposition applies: the kinetic response to an input $q_{1}(t)+q_{2}(t)$ is the sum of the responses to $q_{1}(t)$ and $\mathrm{q}_{2}(\mathrm{t})$ applied separately.

(3) The system is time invariant; its dynamics do not change with time.

After a unit impulsive input-for example, a bolus injection with a unit dose $D=1$-the weighting function is the same as the measured unit impulse response (fig 1).

At a constant rate step input, the system transforms the input into a kinetic response as shown in fig 1 . In this case the unit impulse response may be obtained by a simple deconvolution operation on the step input and the response. Moreover, when both the unit impulse response and the input are known the kinetic response may be calculated by means of a convolution operation on the input and the unit impulse response;

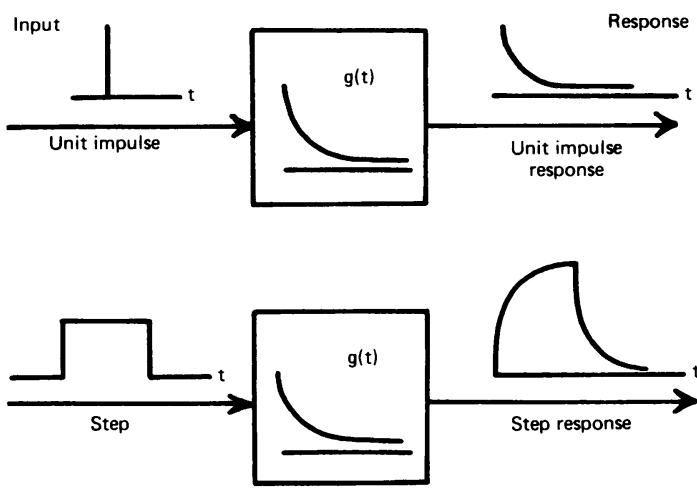

Fig 1 Body as a dynamic system. Weighting function $g(t)$ transforms an input into a kinetic response.

an unknown input may be estimated by means of an inverse operation (deconvolution) on a measured response and the unit impulse response (appendix). In the case of a complicated, non-constant rate of input several methods for numerical deconvolution have been described..$^{7-9}$

In the case of a constant rate of input (q) the deconvolution is substantially simplified (appendix). It may be estimated retrospectively at any postexposure point of time $t_{2}$ with the aid of (i) the unit impulse response $g(t)$ in the observation subsystem and (ii) only one measured concentration in the observation subsystem at $t_{2}$. For a constant step input at $\mathrm{t}<\mathrm{t}_{1}$ the estimated q equals:

$$
q=\frac{C\left(t_{2}\right)}{\int_{t_{2}-t_{1}}^{t_{2}} g(t) \cdot d t}=\frac{C\left(t_{2}\right)}{\left.\operatorname{Area~} g(t)\right|_{t_{2}-t_{1}} ^{t_{2}}}
$$

The estimation of the constant rate of input has now been simplified by means of a measured $C\left(t_{2}\right)$ value divided by an area under the impulse response curve $g(t)$. This area is located just before the time point $t_{2}$ of sampling and has a timelength of $t_{1}$ which equals the duration of exposure. A unit impulse response $g(t)$ is drawn schematically in fig 2 .

The rate of input is defined as the RFI rather than the uptake and it equals the product of the inhaled concentration $\left(C_{1}\right)$ and the functional alveolar ventilation $\left(\dot{\mathrm{V}}_{\mathrm{a}}\right)$ :

$$
\mathrm{RFI}=\mathrm{C}_{\mathrm{l}} \cdot \dot{\mathrm{V}}_{\mathrm{a}}
$$

The functional alveolar ventilation $\left(\dot{\mathrm{V}}_{\mathrm{a}}\right)$ is the virtual air minute volume which completely equilibrates with the mixed venous blood. ${ }^{\text {s }}$ 


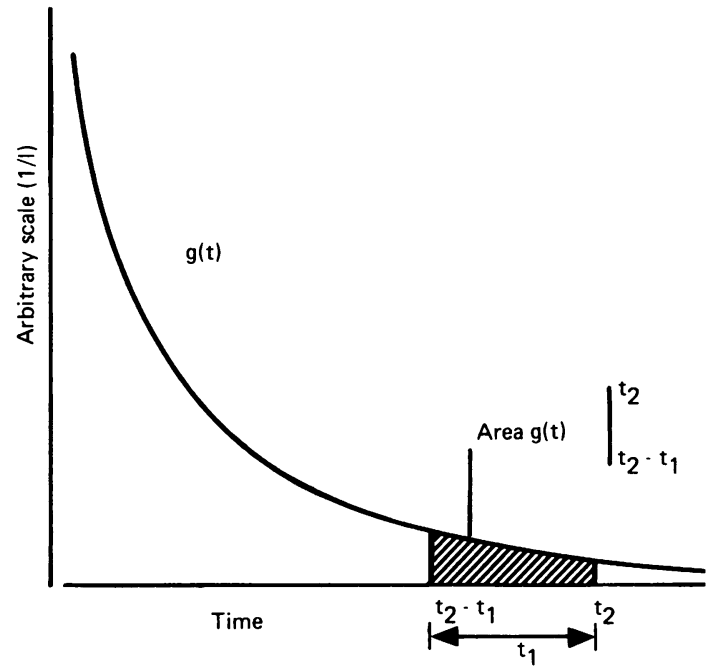

Fig 2 Schematic unit impulse response $g(t)$. Area under $g(t)$ between points of time $\left(t_{2}-t_{1}\right)$ and $t_{2}$ has a time length that equals duration of administration $t_{\text {, }}$.

\section{Methods}

\section{EXPOSURE PROCEDURE}

In a first set of experiments four subjects ( 2 men, 2 women) were exposed twice at rest to PER. The period between the two exposures was about one to two months. A second set was carried out with six subjects ( 3 men, 3 women); each subject was exposed three times simultaneously to PER and TRI at $0 \mathrm{~W}, 30 \mathrm{~W}$, and $65 \mathrm{~W}$ physical exercise. The levels of 30 and $65 \mathrm{~W}$ exercise correspond to light and moderate work load. ${ }^{512}$ The period between the first and the third exposure was two to six months. One man participated
Table 1 Exposures to tetrachloroethene for four subjects at resting conditions; $t_{1}$ : end of exposure, $t_{e}$ : postexposure observation period, and $C_{l}$ : concentration in inhaled air

\begin{tabular}{|c|c|c|c|c|c|c|}
\hline \multirow[b]{2}{*}{ Subject } & \multicolumn{3}{|l|}{ Rest I } & \multicolumn{3}{|l|}{ Rest 2} \\
\hline & $\stackrel{t_{1}}{(\text { min })}$ & $t_{e}(h)$ & $\begin{array}{l}C_{(\mu \mathrm{mol} / \mathrm{l})} \\
\end{array}$ & $\stackrel{t_{1}}{(\min )}$ & $t_{\text {e }}$ & $\begin{array}{l}C_{l} \\
(\mu \mathrm{mol} / \mathrm{l})\end{array}$ \\
\hline $\begin{array}{l}1 \mathrm{~m} \\
2 \mathrm{~m} \\
\text { If } \\
2 \mathrm{f}\end{array}$ & $\begin{array}{l}48 \cdot 2 \\
42 \cdot 7 \\
28 \cdot 7 \\
40 \cdot 3\end{array}$ & $\begin{array}{r}240 \\
70 \\
185 \\
260\end{array}$ & $\begin{array}{l}0.33 \\
0.21 \\
0.34 \\
0.34\end{array}$ & $\begin{array}{l}36 \cdot 8 \\
41 \cdot 1 \\
45 \cdot 8 \\
60 \cdot 2\end{array}$ & $\begin{array}{l}190 \\
170 \\
285 \\
280\end{array}$ & $\begin{array}{l}0.38 \\
0.35 \\
0.35 \\
0.36\end{array}$ \\
\hline
\end{tabular}

in both sets. The subjects were aged $24-40$; the percentage of fat tissue according to the method of Durbin and Wormersly ${ }^{13}$ was $10-26 \%$ for the men and $25-31 \%$ for the women.

Tables 1 and 2 present the exposure data of both sets of experiments. Concentrations in inhaled air ranged from 0.11 to $0.43 \mu \mathrm{mol} / 1(18-71 \mu \mathrm{g} / \mathrm{l})$ for PER and from 0.24 to $1.58 \mu \mathrm{mol} / 1(31-205 \mu \mathrm{g} / \mathrm{l})$ for TRI. Exposure lasted for 29 to 62 minutes. The postexposure period of observation lasted 70-500 hours for PER and 20-310 hours for TRI.

The conditions of exposure, the sampling protocol, and the sampling errors have been described in detail. ${ }^{5}$ The method of exposure and of alveolar air sampling has also been described earlier."

\section{INDIVIDUAL UNIT IMPULSE RESPONSE}

For each subject one of the two or three experiments was taken as a "reference" experiment; the individual impulse response was calculated from $(a)$ the kinetic response in the postexposure period and $(b)$ the rate of input $(\mu \mathrm{mol} / \mathrm{min})$ during exposure. The other one or two experiments were called the "occupational" experiments and were carried out to test the method of retrospective estimation by estimating their "unknown" respiratory input.

Table 2 Three simultaneous exposures to tetrachloroethene and trichloroethene for six subjects at three conditions of rest, $30 \mathrm{~W}$, and $65 \mathrm{~W}$ physical exercise

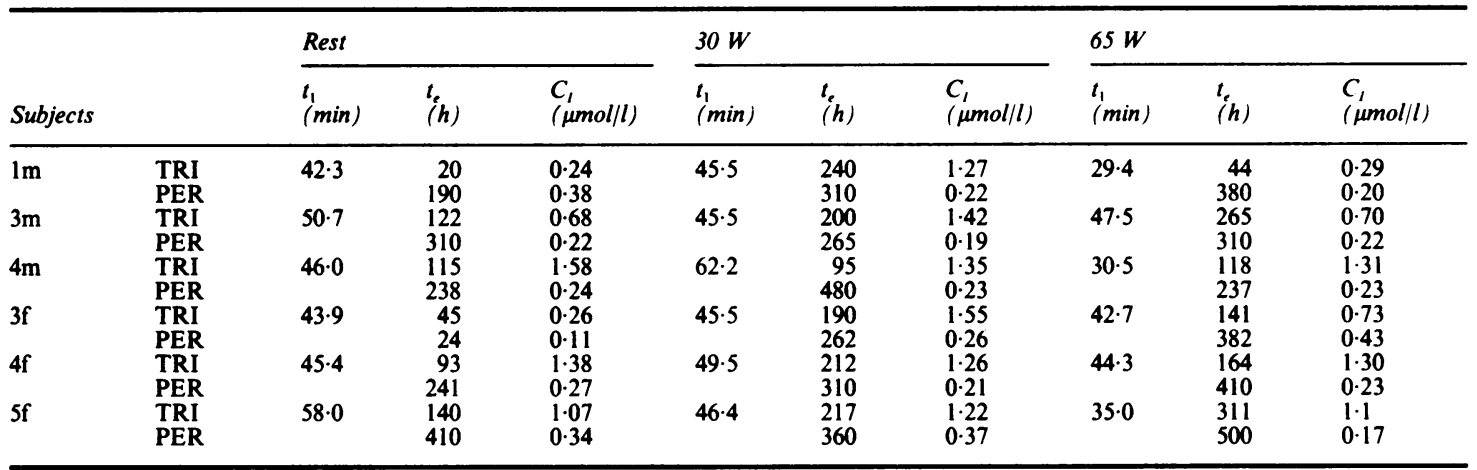

$t_{1}=$ End of exposure.

$\mathrm{t}_{\mathrm{c}}=$ Postexposure observation period.

$C_{1}=$ Concentration in inhaled air. 


\section{KINETIC RESPONSE}

In the reference experiment the kinetic response to a step input in the whole postexposure period $\left(t>t_{1}\right)$ after a step input was expressed by a mathematical multiexponential function (eq 3.2 ; table 3 ). The parameters in this function (coefficients $c(i)$ and exponents $r(i)$ ) were estimated by means of curve fitting the measured data of the alveolar concentration $\left(C_{\text {alv }}\right)$. The following $C_{\text {alv }}$ values were used: (1) all values during the first postexposure day and (2) all values sampled at the subsequent mornings. The $\mathrm{C}_{\mathrm{alv}}$ level at the end of exposure was used to limit the fitted $C_{\text {alv }}$ level at the end of exposure. The morning $C_{\text {alv }}$ values rather than the evening $\mathrm{C}_{\mathrm{alv}}$ were used because of the previous "standardised" sleeping period. The postexposure data were fitted because the weighted residual error around a fitted line was expected to be smaller than during exposure. "The data were fitted by minimising the sum of weighted least squares (eq 3.3; table 3). The variability of the data around the fitted line was expressed by the weighted residual error (WRE) (eq 3.4; table 3). The fitting was carried out with a sequential simplex procedure. ${ }^{14}$

The mathematical expression of the unit impulse response $g(t)$ obtained from a step input (RFI = constant) is given by equation 3.5 (table 3 ).

\section{RATE OF INPUT}

The RFI is given in eq 2 . The experimental determination of $\dot{V}_{a}$ has been described in a previous paper. ${ }^{5}$

The RFI in the occupational experiments by the same subjects was estimated several times independent of each other at different times in the whole postexposure period with the aid of one measured $\mathrm{C}_{\text {alv }}$ value and the impulse response $g(t)((\mathrm{eq} 1)$ and appendix).
Table 3 Equations used to evaluate measured data

\begin{tabular}{|c|c|c|}
\hline $\begin{array}{l}C_{\text {alv }}(t) \\
t \leqslant t_{1}\end{array}$ & $=\sum_{i=1}^{p} c(i)(1-\exp (r(i) t))$ & $(3 \cdot 1)$ \\
\hline $\begin{array}{l}C_{\text {1lv }}(t) \\
t \geqslant t_{1}\end{array}$ & $\left.=\sum_{i=1}^{p} c(i)\left(\exp \left(r(i) t-t_{1}\right)\right)-\exp (r(i) t)\right)$ & $(3 \cdot 2)$ \\
\hline WLS* & $=\sum_{j=1}^{n}\left(\left(y_{j}-\hat{y}_{j}\right) / \hat{y}_{j}\right)^{2}$ & (3.3) \\
\hline WRE* & $=\sqrt{\left.\sum_{j=1}^{n} 1 /(n-2 p) \cdot\left(y_{j}-\hat{y}_{j}\right) / \hat{y}_{j}\right)^{2}} \times 100 \%$ & $(3.4)$ \\
\hline$g(t)$ & $=(1 / R F I) \sum^{p}(-1) c(i) r(i) \cdot \exp (r(i) t)$ & $(3 \cdot 5)$ \\
\hline
\end{tabular}

\section{Results}

\section{COURSE OF ALVEOLAR CONCENTRATION}

Figures $3 \mathrm{a}, 3 \mathrm{~b}$, and 4 show the kinetic response in the alveolar air during and after simultaneous exposure to PER and TRI of subject $4 f$ at rest. These figures are representative of the results of all subjects. Figures 3(a) and (b) show the $\mathrm{C}_{\text {alveq }}$ data relative to the inhaled concentration $\left(C_{l}\right)$ during the first 60 minutes after the onset of a simultaneous exposure to PER and TRI at rest. The $\mathrm{C}_{\text {alv.eq }}$ data are in equilibrium with the concentration in mixed venous blood."

Figure 4 shows the $C_{a l v, e q}$ data relative to $C_{1}$ for PER and TRI in the postexposure period up to 11 days.

The curvefitting according to equation 3.2 resulted in several estimates of the coefficients $c(i)$ and exponents $r(i)$. Table 4 presents the estimated $c(i)$ and $r(i)$ parameters of subject $4 \mathrm{f}$ at rest, 30 , and $65 \mathrm{~W}$ exercise. For an adequate fitting of the $\mathrm{C}_{\mathrm{alv}}$ values corresponding to a minimal sum of weighted least squares (WLS) (eq 3.3), the time course of PER mostly required five
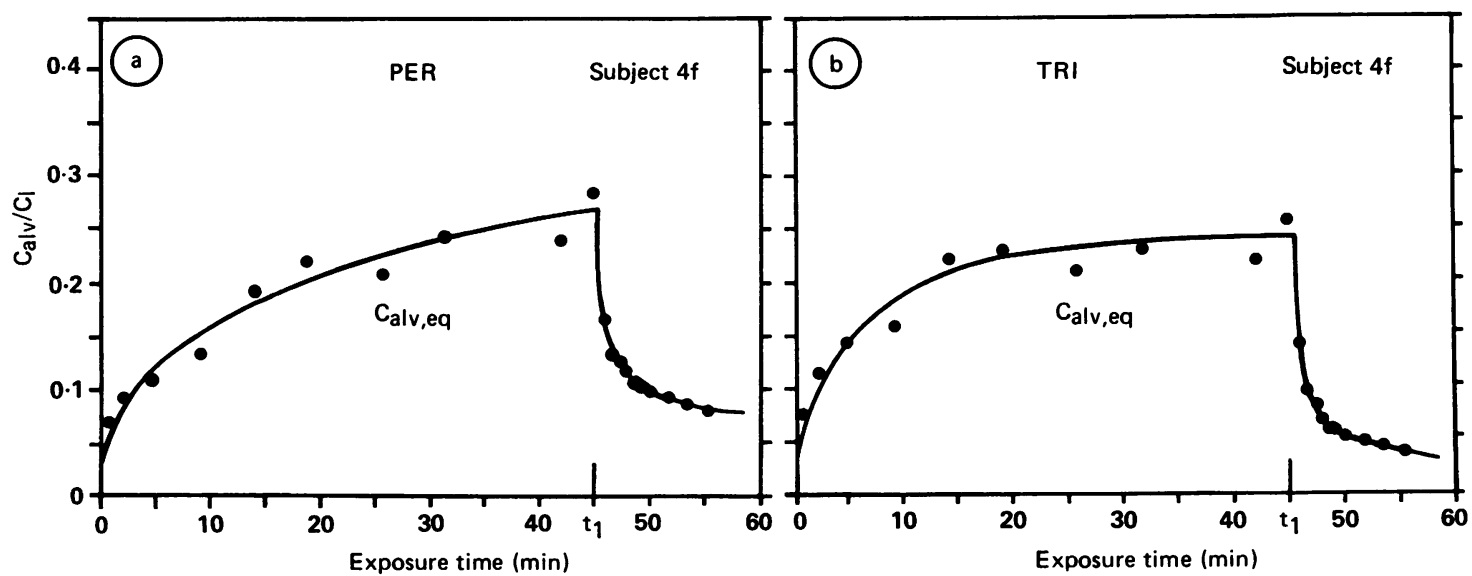

Figs 3a, b Alveolar concentration curves relative to $C_{1}$ up to 60 minutes after onset of exposure for both PER and TRI for subject $4 f$ exposed simultaneously to PER and TRI at rest. $C_{\text {alv. eq }}$ data are in equilibrium with concentration $C_{\text {ven }}$ in mixed venous blood and are obtained after a normal inhalation, five seconds breathholding, and normal exhalation. 
exponential terms $(p=5)$ whereas for TRI only half the cases if our terms were sufficient. In a few experiments with a short postexposure period of 48 hours for TRI three exponential terms were sufficient. For all subjects the average weighted residual error (WRE) (eq 3.4; table 3) was 7\% (5.5-9\%) and $11 \%$ (7$15 \%)$ for PER and TRI respectively. This implies a larger intrasubject variation in the timecourse of TRI than that of PER.

In addition it was observed that for all subjects the $\mathrm{C}_{\mathrm{alv}}$ at awakening (symbol $\Delta$ ) was usually higher than the $C_{\text {alv }}$ before sleeping (symbol $\Delta$ ) (fig 4).

\section{IMPULSE RESPONSE}

Figure 5a presents a semilog presentation of the $g(t)$ function of PER and TRI at rest. In general the $g(t)-$ function of TRI at $t=0$ is higher than that of PER but the decrease was faster. In a period of 100 hours the $g(t)$-functions of TRI and PER dropped about 800000 -fold and 20000 -fold respectively. Figure 5b shows a $\log$-log presentation of the $g(t)$-functions obtained from all experiments at rest, 30 , and $65 \mathrm{~W}$ exercise with subject 4f. The $g(t)$-functions of PER and
TRI at rest are the same as in fig 5a. The individual curves are drawn up to the end of the observation period. The presentation pays extra attention to the first day of observation. It should be noted that in figure $5 b$ the scaling for TRI differs from that of PER by a factor of 10 . The figure shows a much lower $g(t)$ function for TRI when exposure is carried out at rest. After 10-20 hours the $g(t)$-functions of PER are similar irrespective of the level of exercise.

\section{ESTIMATION OF THE RFI}

For each subject the error in the retrospective estimated RFI will be expressed as the ratio estimated/ No true RFI; a ratio $=1.0$ indicates a perfect estimate. Figures 6 and 7 respectively show the individual mean ratios at rest and $65 \mathrm{~W}$ exercise with the use of the $c$ impulse responses obtained from the 0 and $30 \mathrm{~W}$ ? experiments. A mean ratio has been obtained from 0 several $\mathbf{n}$ independent ratios each obtained with a single $\mathrm{C}_{\mathrm{alv}}$ value in a limited postexposure period. The left, middle, and right regions of the figures present the limited periods: 5-60 minutes after exposure, the next hours during the first day, and the subsequent morn- 

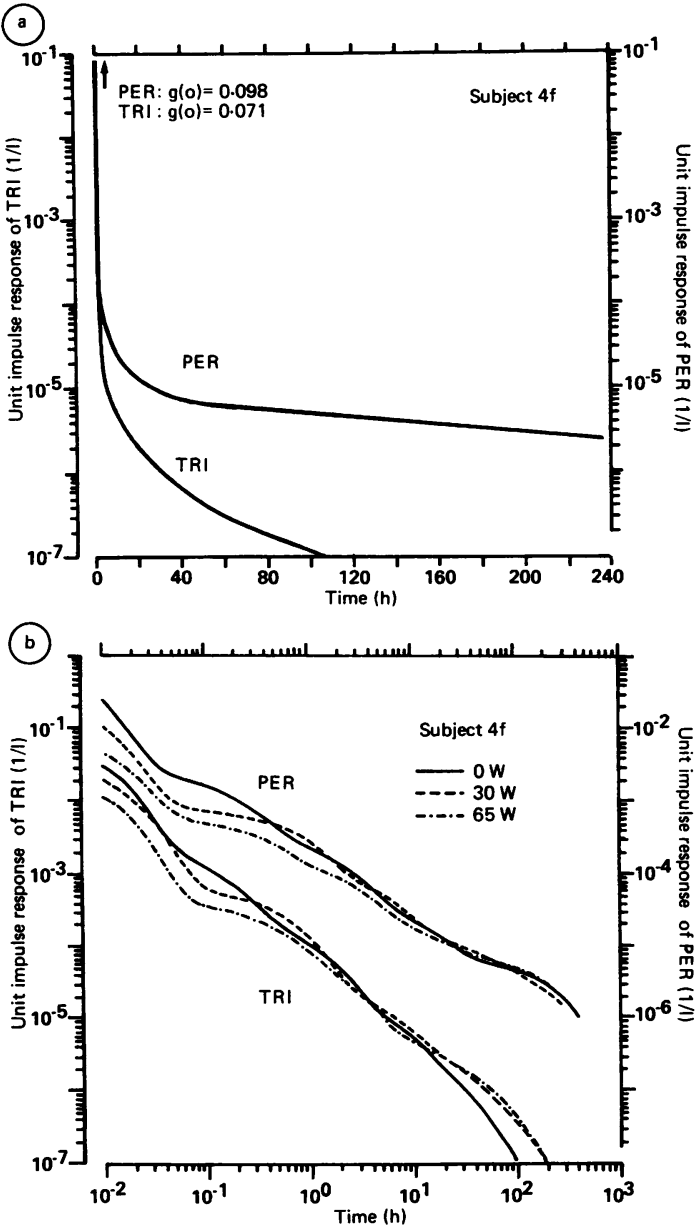

Fig 5 (a) Semilog presentation of unit impulse responses for PER and TRI for subject 4 from experiment at rest. Impulse responses were obtained by deconvolution of constant rate of functional intake (table 6) and fitted time courses; deconvolution yielded eq 3.5 (table 3). Parameters $c(i)$ and $r(i)$ are presented in table 4. (b) Log log presentation of unit impulse responses for TRI and PER for subject 4 from experiments at $0-(-), 30(--)$, and $65(-\cdot) W$ physical exercise. Impulse responses were calculated as described in fig $5 a$.

ings of the following days, respectively.

To estimate the RFI during different levels of exercise, the $30 \mathrm{~W}$ experiment will usually be used as the reference experiment because of the intermediate level of the physical exercise.

RFI OF PER

The functional rate of intake of PER for three of the four subjects belonging to the first set of experiments (twice exposed at rest to PER) was underestimated or overestimated within a $25 \%$ error by using a $C_{\text {alv }}$ value during the first postexposure day (fig 6, left and middle region). Using the subsequent morning concentrations up to 280 hours (right region), estimation with a mean error of less than $10 \%$ for all four subjects was possible. When using only a single morning $\mathrm{C}_{\text {alv }}$ value the $95 \%$ confidence error region of the estimate may be approximated. When using a $\mathrm{CV}=17 \%$ with $\mathrm{n}=$ 12 (subject $2 \mathrm{f}$ ) then for all subjects the $95 \%$ confidence error intervals may be set to $\pm 34 \%$. When using two morning $\mathrm{C}_{\text {alv }}$ values the $95 \%$ confidence error interval reduces to $\pm 24 \%$.

The difference between the durations of exposure did not have a striking effect on the accuracy of the estimated intake. For example, for subject $2 f(\diamond)$ the durations of the reference and occupational experiments were 40 and 60 minutes.

To estimate the RFI during different levels of exercise the use of the morning $C_{\text {alv }}$ data - that is, at least 15 up to 400 hours after exposure-yields a RFI estimate with a mean error within $25 \%$ for the subjects (fig 7a, b; right region). The average of $\mathrm{CV}$ equals $12 \%$ with all subjects and, therefore, the $95 \%$ confidence interval of a single estimate equals about $25 \%$.

For subject $3 \mathrm{f}$ the observation period after the rest exposure was limited to 24 hours because a short visit to a chemical cleaning shop increased the postexposure concentrations about threefold.

One subject showed a systematic underestimation $(30 \%)$ of the RFI at rest because the CV of the RFI estimates was only $7 \%$ for 15 independent estimates (fig 7a, right region). The systematic error probably was due to an error in the "true" RFI during rest because the RFI at $65 \mathrm{~W}$ exercise was estimated reasonably well (fig $7 b$, right region).

The differences in the duration of exposure do not seem to have a striking effect on the accuracy of the intake estimate, although differences in the duration of exposure in a $30 \mathrm{~W}$ and a $65 \mathrm{~W}$ experiment ranges up to twofold (table 2; subject $4 \mathrm{~m}$ ).

To estimate the RFI the $\mathrm{C}_{\mathrm{alv}}$ data obtained in the first postexposure hour (5-60 minutes) appeared not to be preferred for all subjects (left region; fig 7a, b). For both at rest and $65 \mathrm{~W}$ exercise the mean error of the RFI estimate ranges up to $100 \%$ (figs $7 \mathrm{a}, \mathrm{b}$ ).

The next hours during the day of exposure (fig 7a, b); middle region may be a suitable period. The RFI at rest appeared to be estimated well whereas the ability to estimate the RFI at $65 \mathrm{~W}$ exercise seems doubtful.

\section{RFI OF TRI}

The errors in the estimated RFI of TRI for six subjects are presented in fig $7 \mathrm{c}, \mathrm{d}$.

The $\mathrm{C}_{\mathrm{alv}}$ data in the early postexposure period of 
Table 4 Coefficients $c(i)$, exponents $r(i)$, and weighted residual errors (WRE) of subject 4 f obtained from curve fitting of several $n C_{\text {alv }}$ values after a simultaneous exposure to PER and TRI at rest, 30, and $65 \mathrm{~W}$ physical exercise

\begin{tabular}{|c|c|c|c|c|c|c|}
\hline & $P E R$ & $T R I$ & $P E R$ & $T R I$ & \multirow[b]{2}{*}{$P E R$} & \multirow[b]{2}{*}{$T R I$} \\
\hline & \multicolumn{2}{|c|}{$c(i) / C_{1} ; i=1, \ldots, 5$} & \multicolumn{2}{|c|}{$r(i) ; i=1, \ldots, 5$} & & \\
\hline Rest & $\begin{array}{l}0.147 \\
0.079 \\
0.119 \\
0.087 \\
0.346\end{array}$ & $\begin{array}{l}0.164 \\
0.057 \\
0.053 \\
0.020 \\
0.0151\end{array}$ & $\begin{array}{l}-2.45 \\
-0.103 \\
-0.0120 \\
-0.00174 \\
-0.000086\end{array}$ & $\begin{array}{l}-1.55 \\
-0.136 \\
-0.0156 \\
-0.00218 \\
-0.00054\end{array}$ & $\begin{array}{l}\text { No }=31 \\
\text { WRE }=5.5 \% \\
\text { RFI }=1 \mu \mathrm{mol} / \mathrm{min} \\
(166 \mu \mathrm{g} / \mathrm{min})\end{array}$ & $\begin{array}{l}27 \\
6.7 \% \\
5.15 \mu \mathrm{mol} / \mathrm{min} \\
(670 \mu \mathrm{g} / \mathrm{min})\end{array}$ \\
\hline $30 \mathrm{~W}$ & $\begin{array}{l}0.162 \\
0.273 \\
0.250 \\
0.258 \\
0.786\end{array}$ & $\begin{array}{l}0.345 \\
0.20 \\
0.074 \\
0.065 \\
0.052\end{array}$ & $\begin{array}{l}-1.82 \\
-0.0262 \\
-0.0051 \\
-0.00078 \\
-0.000086\end{array}$ & $\begin{array}{l}-1.12 \\
-0.032 \\
-0.0055 \\
-0.00078 \\
-0.00021\end{array}$ & $\begin{array}{l}\text { No }=30 \\
\text { WRE }=8 \% \\
\text { RFI }=2.26 \mu \mathrm{mol} / \mathrm{min} \\
\quad(370 \mu \mathrm{g} / \mathrm{min})\end{array}$ & $\begin{array}{l}25 \\
17.5 \% \\
13.2 \mu \mathrm{mol} / \mathrm{min} \\
(1720 \mu \mathrm{g} / \mathrm{min})\end{array}$ \\
\hline $65 \mathrm{~W}$ & $\begin{array}{l}0.098 \\
0.147 \\
0.293 \\
0.168 \\
1.46\end{array}$ & $\begin{array}{l}0.332 \\
0 \cdot 167 \\
0 \cdot 152 \\
0.192\end{array}$ & $\begin{array}{l}-1.43 \\
-0.051 \\
-0.0067 \\
-0.0010 \\
-0.000091\end{array}$ & $\begin{array}{l}-1.46 \\
-0.036 \\
-0.0067 \\
-0.00036\end{array}$ & $\begin{array}{l}\text { No }=33 \\
\text { WRE }=7 \% \\
\text { RFI }=3.53 \mu \mathrm{mol} / \mathrm{min} \\
\quad(578 \mu \mathrm{g} / \mathrm{min})\end{array}$ & $\begin{array}{l}26 \\
14 \cdot 8 \% \\
20 \cdot 5 \mu \mathrm{mol} / \mathrm{min} \\
(2660 \mu \mathrm{g} / \mathrm{min})\end{array}$ \\
\hline
\end{tabular}

RFI: rate of functional intake.

5-60 minutes are not completely suitable in any subject. For at least three subjects the estimated/true RFI ratio is outside the $1.00-1.25$ range of error (fig 7c, $\mathrm{d}$; left region).

The $\mathrm{C}_{\mathrm{alv}}$ data during one to 15 hours on the day of exposure might be of interest (fig 7c, d; middle region). The RFI at $65 \mathrm{~W}$ exercise for five of the six subjects was estimated within an error of $25 \%$. The corresponding $\mathrm{CV}$ is rather low except for subject $3 \mathrm{f}$. This was caused by a drop in the first $\mathrm{C}_{\text {alv }}$ data which were obtained at

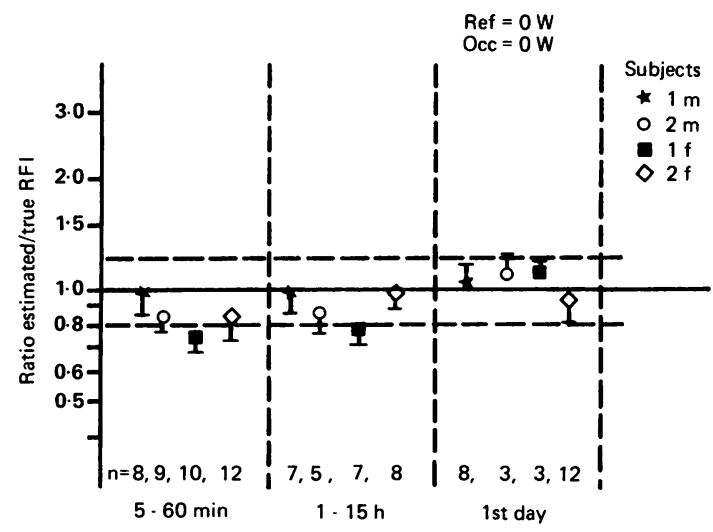

Fig 6 Mean error in retrospectively individual RFI estimates of PER for four subjects. The $25 \%$ underestimates and overestimates are indicated by unbroken lines.

Postexposure period after occupational exposure has been divided into three periods: 5-60 minutes, 1-15 hours, and following days. For each subject mean error and coefficient of variation (vertical lines) were obtained by a number $n$ of independent estimates in three periods. Each subject is presented by his symbol; REF: reference experiment, $O C C$ : occupational experiment. home in the evening hours. This drop was probably due to physical activity during the journey home.

The application of the postexposure morning $\mathrm{C}_{\text {alv }}$ data appears to differ for men and women (fig 7c, d; right region). For the three men the use of $\mathrm{C}_{\mathrm{alv}}$ at the first morning after exposure yielded a RFI estimate within an error of $8-25 \%$ no matter the duration of exposure and the choice of the occupational exposure. For the men the $\mathrm{C}_{\text {alv }}$ measured at the first two mornings delivered acceptable estimates with only a few exceptions. For the three women the RFI estimates appeared to be more sensitive to the level of exercise. The functional intakes at rest were underestimated up to 3.5-fold. Also the errors in the estimation of RFI during $65 \mathrm{~W}$ physical exercise are substantial (fig 7d); subject $4 \mathrm{f}$ is a positive exception.

\section{Discussion}

\section{INDIVIDUAL RFI ESTIMATES}

To estimate the individual RFI a method on an individual basis has been tested. In this study the method applies an experimentally determined unit impulse response and a measured alveolar concentration of the parent compound in the postexposure period after an unknown intake during a variable duration under variable conditions of physical exercise.

The method may only be applied successfully when the intraindividual variability in the kinetics is limited as a function of time (hours, days, months). In our experiments the intraindividual variability in kinetics could be split up into a short and a long term variability. Short term variability became manifest from the variation of the $\mathrm{C}_{\mathrm{alv}}$ data around a theoretical smooth decreasing curve in the postexposure period- 

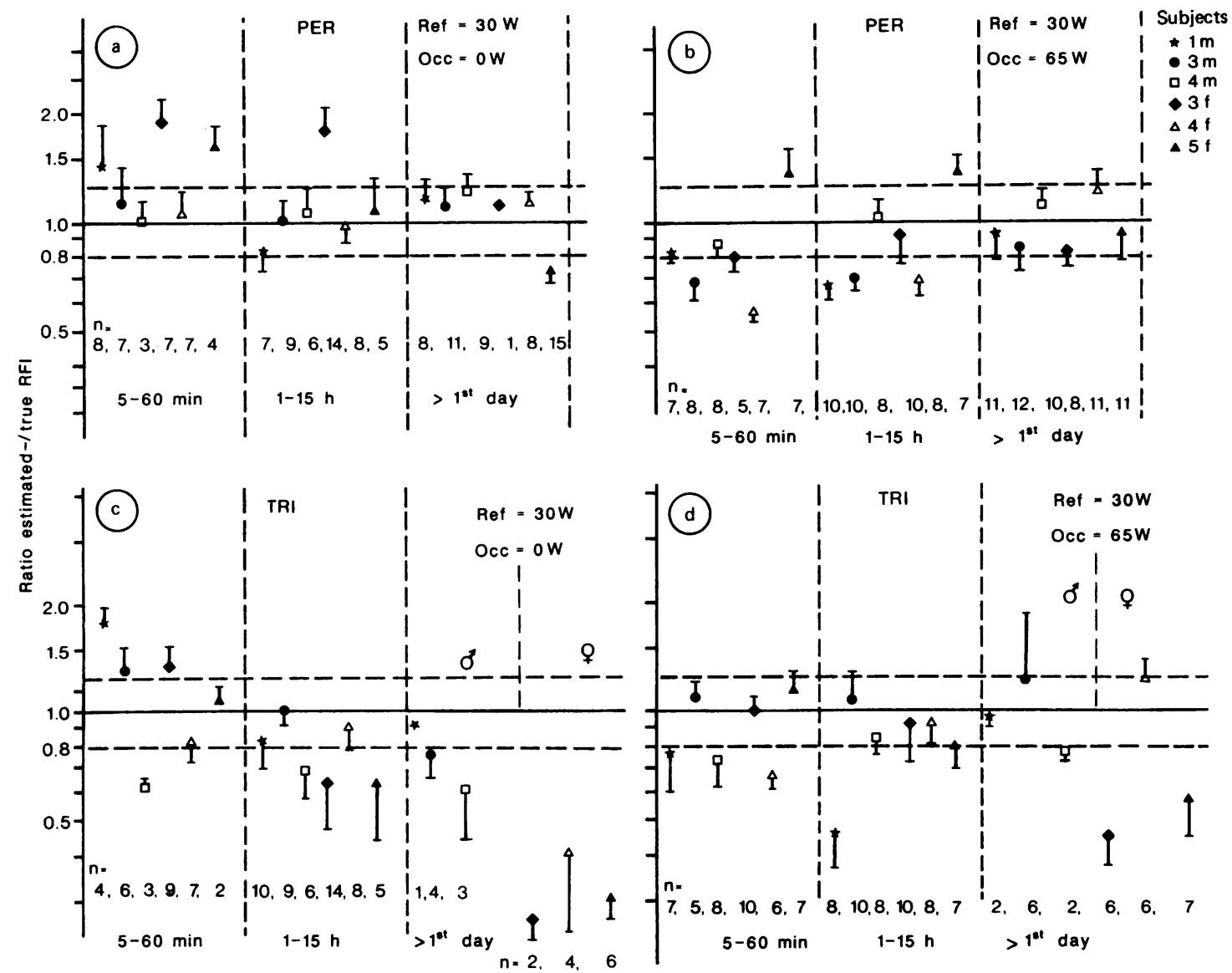

Fig 7 Mean errors in retrospectively individual RFI estimates of PER (7a, b) and TRI (7c,d) for six subjects. The 25\% underestimate and overestimate are indicated by unbroken lines. Postexposure period after occupational exposure has been divided into three periods: 5-60 minutes, 1-15 hours, and following days. For each subject mean error and coefficient of variation (vertical lines) were obtained by a number $n$ of independent estimates in three periods. Each subject is presented by his symbol; REF: reference experiment, OCC; occupational experiment.

for example, the increase of the alveolar concentration during the night (fig 4). The long term intraindividual variability may be shown by intercomparison of the unit impulse responses in each subject exposed two or three times (fig 5b). This long term intraindividual variability became manifest from the accuracy in the retrospective estimates of the RFI with the use of an about one to six months earlier determined impulse response. In fact the errors in the RFI estimates may also be partly explained by the short term variability of the $\mathrm{C}_{\mathrm{avv}}$ data in the occupational experiment. Both the short and long term intraindividual variability will be considered in more detail in a further paper (Opdam, in preparation).

For PER it may be concluded that the measured $C_{\mathrm{alv}}$ values in the postexposure period-that is, from five minutes up to about 300 hours-permits the estimation of the individual RFI ( $\mu \mathrm{mol} / \mathrm{min})$ with an error of less than $25 \%$ for all subjects when resting conditions during the reference and occupational exposure (fig 6 ). When using only one $\mathrm{C}_{\mathrm{alv}}$ value irrespective of the time of sampling in the whole five minutes-300 hours postexposure period, the RFI estimate has a $95 \%$ confidence error interval of about $\pm 35 \%$. Using two $\mathrm{C}_{\text {alv }}$ values the mean RFI estimate will have a confidence interval of $\pm 25 \%$.

At different levels of exercise $(0-65 \mathrm{~W})$ during exposure, the use of a $\mathrm{C}_{\mathrm{alv}}$ value in the postexposure period beyond the day of exposure is to be preferred. The individual RFI of PER during rest and $65 \mathrm{~W}$ 
exercise may be estimated with a mean error of less than $25 \%$ when using the impulse response obtained at the $30 \mathrm{~W}$ experiment and the $\mathrm{C}_{\mathrm{alv}}$ values in the range of 15-500 hours after exposure (fig 7a, b). When using only one postexposure $\mathrm{C}_{\text {alv }}$ value the RFI estimate for an individual subject has a $95 \%$ confidence interval of about $\pm 25 \%$ whereas the use of two $C_{\text {alv }}$ values the confidence error interval of the mean RFI estimate decreases to $\pm 18 \%$.

The level of exercise plays a minor part in the application of the method. Nevertheless, during one to 15 hours after exposure a $\mathrm{C}_{\text {alv }}$ value in combination with the $30 \mathrm{~W}$ impulse response permits an estimate of the PER intake during rest more accurately than during the $65 \mathrm{~W}$ exercise (fig 7a, b).

For some subjects the RFI at rest seems to be overestimated in the postexposure period up to 15 hours whereas the intake at $65 \mathrm{~W}$ seems to be underestimated when using the $30 \mathrm{~W}$ impulse response (fig 7a, b; left region). From a kinetic point of view these errors may be explained by the blood perfusion in subcutaneous fat. The blood flow both through muscles and subcutaneous fat increases with increasing exercise. It is well known, however, that by contrast with the muscle flow, the subcutaneous flow may increase not gradually but abruptly. For lipophilic solvents such as PER the change of flow in subcutaneous fat are reflected in the concentration in the blood; an increased flow will also remain during some time after exercise and results in a decreased blood concentration relative to the intake. The effect of exercise may also be in the impulse responses of PER which, in fact, equal the $\mathrm{C}_{\text {alv }}$ after a bolus input (fig 5b). For some subjects the subcutaneous flow may increase only above $30 \mathrm{~W}$ exercise and, therefore, the 0-15 hours after exposure will be best suited to estimate the RFI at rest with the $30 \mathrm{~W}$ impulse response (fig 7a, subjects $\bullet, \square, \triangle$ ). An increased subcutaneous bloodflow at $30 \mathrm{~W}$ may yield an overestimation of the RFI at rest (fig 7a, subjects *, $\bullet, \Delta$ ). In the $0-15$ hours after exposure the RFI at $65 \mathrm{~W}$ is underestimated for almost all subjects (fig 7b); the subcutaneous bloodflow at $65 \mathrm{~W}$ seems to be increased substantially for most subjects and, therefore, the $\mathrm{C}_{\text {alv }}$ relative to the intake has been decreased.

For TRI the accuracy of the individual RFI estimates depends on the point of time of alveolar sampling, the level of physical exercise during exposure, and the sex.

During the day of exposure the one to 15 hours postexposure period appears to be suitable to estimate the individual RFI of TRI (fig 7c, d; middle region). By contrast with PER, the RFI at $65 \mathrm{~W}$ exercise may be estimated more accurately than the RFI at rest. The $\mathrm{C}_{\text {alv }}$ values during the one to 15 hours permit. an estimate of the RFI at $65 \mathrm{~W}$ exercise within a mean error of $25 \%$ for five of six subjects. The average CV equals $17 \%$ for the subjects and, therefore, when using ? only one $\mathrm{C}_{\text {alv }}$ value the $95 \%$ confidence interval of the $\vec{F}$ RFI equals $\pm 34 \%$. In general the CV value of TRI exceeds that of PER (fig 7) and may be partly explained with the WRE around the postexposure fitted curve of the occupational experiment (table 4). The postexposure $C_{\text {alv }}$ decrease of TRI seems to be more sensitive to normal daily physical activity or changes in rate of metabolism, or both.

Differences related to the sex of the subject are particularly evident when the postexposure period exceeds 15 hours. The $\mathrm{C}_{\text {alv }}$ value on the first morning after exposure permits an estimate of the RFI within an error of $20 \%$ in the men (fig 7c, d). In the women, however, the RFI estimates showed substantial errors with the use of $\mathrm{C}_{\text {alv }}$ data beyond the day of exposure. For women the intraindividual variability in the kinetics of TRI appeared to be substantial in this period.

In general, during the whole postexposure period the $30 \mathrm{~W}$ impulse response is more suited to estimate the TRI intake during a $65 \mathrm{~W}$ exposure than during rest. Particularly in the women, the RFI at rest has been underestimated and, therefore, the kinetics in $₫$ women seem to be more affected by exercise during exposure than in men. For both men and women the $\mathrm{C}_{\mathrm{alv}}$ data relative to the intake were low and as a consequence the not exhaled fraction and thus the metabolised fraction of the intake was shown to be high at rest. ${ }^{5}$ In the women the substantial errors in the RFI estimates at rest cannot be explained only by a $\frac{9}{3}$ high metabolised fraction. We have no explanation based on local blood flows through fatty tissues and the metabolised fraction.

For highly metabolising solvents the impulse responses of the metabolites may be more suited to estimate the RFI than the parent compound in exhaled air. For TRI the metabolites trichloroethanol (TCE) and trichloroaceticacid (TCA) will be used in future work.

\section{A NON-INDIVIDUAL METHOD TO ESTIMATE THE} RFI

To show the usefulness of the individual method a $N$ non-individual method has also been used. This $N$ method uses the impulse response of one subject to 0 estimate the RFI of PER and TRI of the other $\omega$ subjects. Particularly during the postexposure period beyond the day of exposure the limitations of the approach became manifest. For PER the average error $\mathbb{\Phi}$ in the estimate of RFI at rest for eight subjects was $3 \%$ with CV $=33 \%$. For three subjects the RFI was estimated with about $50 \%$ error. The estimations were $\frac{}{\sigma}$ carried out with the impulse response at rest of subject $4 f$ (fig $5 b$ ). For TRI when using only the $C_{\text {alv }}$ value at $\Omega$ 
the first morning the average error in the estimates of RFI at $30 \mathrm{~W}$ exercise for five subjects was $25 \%$ with $\mathrm{CV}=58 \%$. For two female and one male the RFI was estimated with about $70 \%$ error. The estimations were carried out with the $30 \mathrm{~W}$ impulse response of subject $4 f$ (fig $5 b$ ).

Biological monitoring of occupational exposure to PER established a relation between external exposure and exhaled air for a group of workers. ${ }^{4}$ For an individual one exhaled air sample permitted an estimate of the average inhaled concentration with a $95 \%$ confidence interval of $\pm 50-80 \%$.

\section{SIGNIFICANCE FOR BIOLOGICAL MONITORING OF} SOLVENTS

In biological monitoring the use of the unit impulse response appears useful in estimating the individual functional rate of intake and indirectly the actual respiratory uptake. Sampling of the parent compound or of one or more metabolites in exhaled air, urine, or blood, or both, may be applied. Furthermore, the time of sampling is not fixed. The principle of estimating the RFI during a single exposure remains valid at repeated exposures as in industry.

To establish the individual impulse response of each worker is impossible in practice. In fact, for all workers an average impulse response may be used only if the interindividual variability in the kinetics is small. Therefore, knowledge is needed about the magnitude of the interindividual variability in the impulse response of the solvents or their metabolites, or both. In addition, differences in concentrations in the blood may then be predicted among workers at the same level of intake; these differences again may correspond to differences in health risks. Controlled exposure experiments appear to be well suited to investigate the intersubject variation between men and women aged 20 to 65 . At pre-employment such a measurement on the individual level offers the opportunity to discover subgroups with similar impulse responses. This knowledge may be extrapolated to the field of biological monitoring and ultimately to standard setting.

To assess the reliability of extrapolation from the impulse responses in the controlled experiments to occupational exposure, one should realise that only exposure with a limited duration takes place under controlled conditions. Combined exposure in practice may alter the impulse response but to what extent is not yet known. Combined exposure not only due to working conditions but also due to lifestyle-for example, consumption of alcohol-may be a serious problem for an adequate estimation of the RFI. In controlled experiments, however, the use of impulse responses enables us to study quantitatively the varia- tions in the kinetics and the consequences for the RFI estimates.

Furthermore, the experiments increase insight into individual variability in kinetics and consequently in the toxic burden in critical organs, which again reflects the actual health risk.

This study was supported by the Ministry of Social Affairs and Employment. We are grateful to E J Brouwer and P M Gielesen for their experimental work and Dr A C Monster, Professor Dr J M van Rossum, and Professor Dr R L Zielhuis for a critical review of the manuscript.

\section{Appendix}

In linear system dynamics the input and response are related by a convolution integral. When the input $q\left(t^{\prime}\right)$ is applied during a period up to $t_{1}$ the integral can be written as:

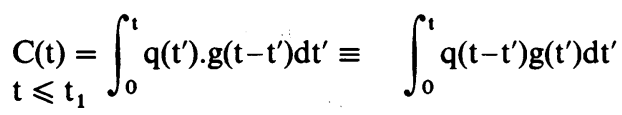

and

$$
\begin{aligned}
& C(t)=t_{1} \\
& t
\end{aligned} \int_{0}^{t_{1}} q\left(t^{\prime}\right) \cdot g\left(t-t^{\prime}\right) d t^{\prime} \equiv \int_{t_{-t_{1}}}^{t} q\left(t-t^{\prime}\right) g\left(t^{\prime}\right) d t^{\prime}
$$

The weighting function $g(t)$ - that is, unit impulse response - of the system weights past values of the input to give the present value of $C(t)$.

In table 5 general equations are summarised. Table 6 shows the mathematical expressions of the kinetic response and the unit impulse response with the assumption that $\mathrm{C}(\mathrm{t})$ is described by a sum of exponentials. The use of exponential terms, which may suggest compartments, are not required. Any function of time may be used-for example, time power functions often seem to be able to fit the data adequately. ${ }^{15}$

The input $q(t)$ may be an impulse or a step input.

Table 5 Response in the output system as a convolution of the unit impulse response $g(t)$ and a rate of input $q(t)$ both at an impulse input and at a step input with duration $t_{1}$

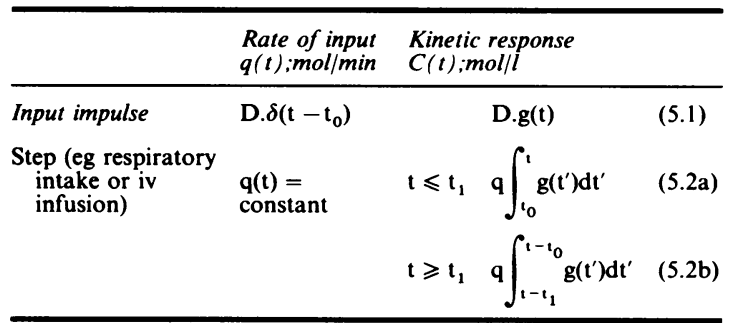


Table 6 Unit impulse response deduced from deconvolution of the rate of input $q$ and the output response $C(t)$. The kinetic response is described as a sum of exponential terms*

\begin{tabular}{|c|c|c|c|}
\hline Input & $\begin{array}{l}\text { Kinetic response } \\
C(t)(\text { mollll }) \dagger\end{array}$ & $\begin{array}{l}\text { Unit impulse response } \\
g(t)(I / l)\end{array}$ & Remarks \\
\hline $\begin{array}{l}\text { Impulse } \\
\text { input }\left(t_{0}=0\right)\end{array}$ & $\begin{array}{l}\Sigma c(i) \cdot \exp (r(i) \cdot t) \\
\Sigma c(i)=D / V_{1}\end{array}$ & $\Sigma c(i) \exp (r(i) \cdot t) / D$ & $g(t)=C(t) / D$ \\
\hline $\begin{array}{l}\text { Step input } \\
q=\text { constant } \\
q \neq \text { Constant }\end{array}$ & $\begin{array}{l}\Sigma c(i) \cdot(1-\exp (r(i) \cdot t)) \\
t<t_{1} \\
\Sigma c(i) \cdot\left(\exp \left(r(i) \cdot\left(t-t_{1}\right)\right)-\exp (r(i) \cdot t)\right) \\
t>t_{1}\end{array}$ & $\Sigma(-1) \cdot c(\mathrm{i}) \cdot \mathrm{r}(\mathrm{i}) / \mathrm{q} \cdot \exp (\mathrm{r}(\mathrm{i}) \cdot \mathrm{t})$ & $\underset{t<t_{1}}{g(t)}=C(t) / q$ \\
\hline
\end{tabular}

*The use of exponentional terms is not a priori required; negative power functions of time may be quite useful. ${ }^{15}$

+Curve fitting of the $C(t)$ data gives the number of exponential terms $(p=1, . .5)$ needed for an adequate fitting. $D=D o s e s$ at an impulse input, $V_{1}=$ distribution volume of the input subsystem.

†Deconvolution may be obtained by a numerical algorithm ${ }^{7-9}$ or by Laplace transformation.

\section{IMPULSE INPUT}

The amount of the compound is administered instantaneously into an input system-for example, the blood. It is assumed that this amount $D$ at point $t_{0}$ is distributed at once in the whole distribution volume $\left(V_{1}\right)$ of this subsystem; this results in a concentration $\mathrm{D} / \mathrm{V}_{1}$ at $\mathrm{t}_{0}$. After the impulse input the response $\mathrm{C}(\mathrm{t})$ that is, the concentration in the blood-is proportional to the weighting function $g(t): C(t)=D \cdot g(t)$ (table 5). After a unit impulse $(D=1)$ the weighting function equals the unit impulse response.

\section{STEP INPUT}

At a constant rate of input the response is simply the integral of the unit impulse response and the convolution integral is simplified substantially (eqs 5.2). Nonconstant inputs often cause problems to evaluate analytically the deconvolution. Even if $\mathrm{C}(\mathrm{t})$ and $\mathrm{g}(\mathrm{t})$ are known analytically, in general the deconvolution cannot be carried out analytically. In this respect numerical deconvolution techniques appear to be suitable. ${ }^{7-9}$

\section{References}

1 Gompertz D. Solvents-the relationship between biological monitoring strategies and metabolic handling: a review. Ann Occup Hyg 1980;23:403-10.

2 Engström K, Husman K, Pfäfi P, Riihimäku V. Evaluation of occupational exposure to xylene by blood, exhaled air and urine analysis. Scand J Work Environ Health 1978;4:114-21.

3 Veulemans H, van Vlem E, Janssens H, Masschelein R. Exposure to toluene and urinary hippuric acid excretion in a group of rotogravure printing workers. Int Arch Occup Environ Health 1979;44:99-107.

4 Monster A, Regouin-Peeters W, van Schijndel A, van der Tuin J. Biological monitoring of occupational exposure to tetrachloroethene. Scand J Work Environ Health 1983;8:273-81.

5 Opdam JJG. The respiratory input in inhalation experiments. $\mathrm{Br} J$ Ind Med 1989;46:145-56.

6 Bassingthwaighte JB. Blood flow and diffusion through mammalian organs. Stochastic methods used for chemical reactor analysis are readily applicable to complex biologic systems. Science 1970;167:1347-53.

7 Vaughan DP, Dennis M. Mathematical basis of the "point-area" deconvolution method for determining in vivo input functions. J Pharm Sci 1978;67:663-7.

8 Cutler DJ. Numerical deconvolution by least squares: use of prescribed input functions; use of polynomials to represent input functions. $J$ Pharmacokin Biopharm 1978;6:227-41, 242-63.

9 Vent Pedersen P. Novel deconvolution method for linear pharmacokinetic systems with polyexponential impulse response. J Pharm Sci 1980;3:312-8.

10 van Rossum JM, Burgers J, van Lingen G, de Bie J. Pharmacokinetics: a dynamic system approach. Trends in Pharmacological Sciences 1983;4:27-30.

11 Opdam JJG, Smolders JFJ. Alveolar sampling and fast kinetics of tetrachloroethene in man. I. Alveolar sampling. $\mathrm{Br} \mathrm{J}$ Ind Med 1986;43:814-24.

12 Astrand I. Effect of physical exercise on uptake, distribution and elimination of vapors in man. In: Fiserova-Bergerova V, ed. Modelling of inhalation exposure to vapours: uptake, distribution and elimination. Vol 2. Boca Raton, Florida: CRC Press, 1983:107-30.

13 Durmin IVGA, Womersley J. Body fat assessed from total body density and its estimation from skinfold thickness. Br J Nutr 1974;32:77-83.

14 Nelder JA, Mead R. A simplex method for function minimization Computer Journal 1965;7:308-13.

15 Wise ME. Scientific commentary; negative power functions of time in pharmacokinetics and their implications. $J$ Pharmacokin Biopharm 1985;13:309-46. 